\title{
PREIMAGES OF METRIC SPACES
}

\author{
BY G. M. REED AND P. L. ZENOR
}

Communicated by W. Wistar Comfort, March 14, 1974

The authors would like to dedicate these results to their former teachers Ben Fitzpatrick and D. R. Traylor.

In 1937, F. B. Jones showed [5] that under the assumption of the continuum hypothesis each normal, separable Moore space is metrizable. Since that time, Jones' "normal Moore space conjecture," i.e., the conjecture that each normal Moore space is metrizable, has been one of the most tantalizing open questions in general topology. Furthermore, other than R. H. Bing's result of 1951 [1] that each collectionwise normal Moore space is metrizable, the only positive results on this particular problem have depended on various set theoretic assumptions. In fact, the work of Bing [1], [2], R. W. Heath [7], J. H. Silver, and F. D. Tall [9], [10] has shown that the metrizability of normal, separable Moore spaces, as well as several related conjectures, are actually independent of set theory.

It is the purpose of this announcement to give a positive result concerning the metrization of normal, locally compact Moore spaces which requires no set theoretic assumptions. B. Fitzpatrick and D. R. Traylor showed [3] that if there exists a normal, separable, nonmetrizable Moore space, then there exists one that is also locally compact. Also, W. G. Fleissner has recently shown [4] that it is consistent that each normal, locally compact Moore space be metrizable. Thus, it is now known that the metrizability of normal, locally compact Moore spaces is independent of set theory. However, the question has often been raised (Jones [6] and Traylor [11], for example) as to whether each normal, locally compact, locally connected Moore space is metrizable. This question seems to be a good candidate for yet another independence result, since it follows from known results that it is consistent to claim the existence of a normal, complete, locally connected, nonmetrizable Moore space. But this is not the case, for in Theorem 1 the authors show, without any set theoretic assumptions other than the axiom of choice, that each normal, locally compact, locally connected Moore space is metrizable.

REMARK. For convenience, the results given below are stated entirely within the context of Moore spaces. However, in the paper to which this announcement refers, the authors obtain results which are considerably

AMS (MOS) subject classifications (1970). Primary 55E30, 55E35. 
more general. In addition, new mapping concepts not mentioned here are defined and investigated.

LEMMA 1. Each Moore space of cardinality $\leqq$ c has a countable separating open cover.

LEMMA 2. Each normal Moore space of cardinality $\leqq c$ admits a continuous one-to-one map onto a subspace of Hilbert space.

LEMMA 3. (Follows from results in [12].) Each locally compact, locally connected Moore space which admits a continuous one-to-one map onto a metric space is metrizable.

LEMma 4. If $S$ is a Moore space in which each point is contained in a connected open set of cardinality $\leqq$, then each component of $S$ has

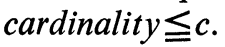

THEOREM 1. Each normal, locally compact, locally connected Moore space is metrizable.

\section{REFERENCES}

1. R. H. Bing, Metrization of topological spaces, Canad. J. Math. 3 (1951), 175-186. MR 13, 264.

2. - A translation of the normal Moore space conjecture, Proc. Amer. Math. Soc. 16 (1965), 612-619. MR 31 \#6201.

3. B. Fitzpatrick, Jr. and D. R. Traylor, Two theorems on metrizability of Moore spaces, Pacific J. Math. 19 (1966), 259-264. MR 34 \#3535.

4. W. G. Fleissner, Normal Moore spaces in the constructible universe (to appear).

5. F. B. Jones, Concerning normal and completely normal spaces, Bull. Amer. Math. Soc. 43 (1937), 671-677.

6. - Review \#3535 (of reference 3 above), Math. Reviews 34 (1967), 643.

7. R. W. Heath, Screenability, pointwise paracompactness, and metrization of Moore spaces, Canad. J. Math. 16 (1964), 763-770. MR 29 \#4033.

8. R. L. Moore, Foundations of point set theory, rev. ed., Amer. Math. Soc. Colloq. Publ., vol. 13, Amer. Math. Soc., Providence, R.I., 1962. MR 27 \#709.

9. F. D. Tall, Set-theoretic consistency results and topological theorems concerning the normal Moore space conjecture and related problems, Thesis, University of Wisconsin, Madison, Wis., 1969.

10.,$- P$-points in $\beta N-N$, normal nonmetrizable Moore spaces, and other problems of Hausdorff, Proc. 1972 Pittsburgh Internat. Conference on General Topology (to appear).

11. D. R. Traylor, On normality, pointwise paracompactness and the metrization question, Proc. 1967 Arizona State Univ. Topology Conf., Tempe, Arizona, 1968, pp. 286-289.

12. P. L. Zenor, Spaces with regular $G_{\delta^{-}}$diagonals, Proc. Third Prague Top. Symp. $1971,471-473$.

Department of Mathematics, Ohio University, Athens, Ohio 45701

Department of Mathematics, Auburn University, Auburn, Alabama 36830 